$\xi_{p=}$

\title{
Is the expansion of universe accelerating or the Photons decelerating?
}

\author{
Hasmukh K. Tank \\ Indian Space Research Organization, 22/693 Krishna Dham-2 Ahmedabad-380015 India \\ E-mail:tank.hasmukh@rediffmail.com
}

\begin{abstract}
Astronomical observations of the cosmological red-shift are currently interpreted in terms of 'expansion of universe' and 'accelerated-expansion of the universe', at the rate of $H_{0} c$; here $H_{0}$ is Hubble's constant, and $\mathrm{c}$ is the speed of light. Whereas a straight-forward derivation presented here suggests that: rather it is the photon which is decelerating, at the rate of $H_{0} c$. Such a deceleration of photons can be caused by virtual electrons, positrons and pi-mesons, contained in the extra galactic quantum vacuum, because: they do have gravitational-acceleration of the same order as $H_{0} c$ at their "surfaces"; or by decay of a photon into a lighter photon and a particle of mass $h H_{0} / c^{2}$. Tired-light interpretations of the cosmological red-shift' were so far considered as not compatible with the observations of 'time-dilation of super-novae light-curves'; so in a paper titled: "Wave-theoretical insight into the relativistic 'lengthcontraction' and 'time-dilation of super-novae light-curves"' (Tank, Hasmukh K. 2013), it has been already shown that any mechanism which can cause 'cosmological red-shift' will also cause 'time-dilation of super-novae light-curves'. Therefore, we now need not to remain confined to the Big-Bang model of cosmology.
\end{abstract}

Keywords: Cosmology; Cosmological Red-Shift; Accelerated Expansion of the Universe; Steady-State-Cosmology.

\section{Introduction}

E.P. Hubble's observations of 'cosmological red-shift', and subsequent observations till today, are currently interpreted in terms of 'expansion of the universe' and accelerated-expansion of the universe at the rate $H_{0} c$. Whereas a straight-forward derivation presented here suggests that: rather it is the photon which is decelerating, at the rate of $H_{0} c$. Tired-light interpretations of a cosmological red-shift' were so far considered as not compatible with the observations of 'time-dilation of super-novae light-curves'; so in a paper titled: "Wave-theoretical insight into the relativistic 'lengthcontraction' and 'time-dilation of super-novae light-curves" (Tank, Hasmukh K. 2013) it has been already shown that any mechanism which can cause 'cosmological red-shift' will also cause 'time-dilation of super-novae light-curves'. Therefore, we now need not to remain confined to the Big Bang model of cosmology.

Martin Lopez-Corredoira (2014) applied Alcock- Paczynski cosmological test to six candidate cosmological models; and reached a conclusion that only two of them, namely lambda-CDM and static universe with tired light interpretation of the cosmological red-shift, fit the data.

Cosmologists have accepted the 'space-expansion- interpretation' of the 'cosmological red-shift', as it matches with Einstein's General Theory of Relativity (GR), which predicted 'expansion of space'. This author has been arguing that 'expansion of space' of GR is a 'mathematical terminology' not a physical phenomenon; because: as per GR, if the space between the galaxies is expanding, but the space within the galaxy is not doing so, as a galaxy is a gravitationally-bound structure, then what happens at the boundary of the galaxy? Such uneven expansion of glass would break the glass, and may tear off the space, if expansion-of-space were a physically-real phenomenon. Recently, Ling Jun Wang, (2014) in an article, "On the Flatness of Space-time" has rigorously proven that curvature and expansion of space-time are mathematical entities, not the physically real curvature or expansion of space. So, the static universe with tired-light explanation has remained the only candidate. In this paper 'cosmological red-shift' is explained in terms of deceleration of photons caused by virtual electrons and pi-mesons contained in the extra galactic quantum vacuum. And, as supportive evidence, it is shown here that virtual electrons and pi-mesons do have gravitational-acceleration of the order $H_{0} c$ at their "surfaces." As a further supportive evidence for this proposal, it is shown that even the space-probes Pioneer-10, Pioneer11, Galileo and Ulysses too, did decelerate at the same rate $H_{0} \mathrm{c}$ (Anderson J.D. at all 1998).

\section{The derivations}

i) It is currently believed that the expansion of the universe is getting accelerated at the rate $H_{0} c$. The following derivation suggests that rather it is the cosmologically red-shifting photon, which is decelerating at the same rate. The linear part of the cosmological red-shift is expressed as:

$z_{c}=h \Delta f / h f=H_{0} D / c$

That is, the loss in energy of the photon, at a distance $D$, is:

$h \Delta f=\left(h f / c^{2}\right)\left(H_{0} c\right) D$ 
That is, the loss in energy of the photon at a distance $D$ is equal to its "mass" times the acceleration $\left(H_{0} c\right)$ times the distance $D$. Whether the expansion of the universe is accelerating, is still a hypothesis; whereas the cosmologically red-shifting photon is decelerating at the same rate $\left(H_{0} c\right)$, as found here, is an experimentally observed fact.

The mechanisms, which can cause such a deceleration of the photons, can be explained as follows:

a) The inter-galactic space is not a void extension of nothingness. As per quantum mechanics, pairs of virtual particle-andantiparticles keep on coming in existence for small durations of time permitted by 'Heisenberg's uncertainty principle'. There are many pairs of virtual particles always present in the path of inter-galactic photons. The gravitational-acceleration at the "surface" of these particles is of the order of $H_{0} c$ as shown blow: So all these virtual particles, bit by bit, keep on causing 'gravitational red-shift' in the inter-galactic photons.

Gravitational acceleration at the "surface" of the electron:

$a_{e}=G m_{e} /\left(e^{2} / 2 m_{e} c^{2}\right)^{2}=H_{0} c$

Gravitational acceleration at the surface of the pi-meson can also be derived from Steven Weinberg's formula, that: $m_{p i}=\left[h^{2} H_{0} / c G\right]^{1 / 3}$

i.e. $G m_{p i} /\left(h / m_{p i} c\right)^{2}=H_{0} c$

Even the accelerations at the "surface" of the nucleus-of-atom, the globular-clusters, the spiral-galaxies, and the galactic-clusters too, are of the order of $H_{0} c$ as Siveram C. (1994) has numerically found:

i) For a typical atomic nucleus of mass $m_{n},(\mathrm{~A}=150)$

$a=G m_{n} / r_{n}^{2} \sim 1.0 \times 10^{-10} \mathrm{~m} / \mathrm{s}^{2}$

ii) For a globular cluster of mass $10^{6}$ solar-masses and radius $R_{g}=$ $100 \mathrm{pc}$,

$a=G M_{g} / R_{g}{ }^{2} \sim 10^{-10} \mathrm{~m} / \mathrm{s}^{2}$

iii) For a spiral galaxy of mass $M_{\text {gal }}=10^{12}$ solar-masses and radius $R_{\text {gal }}=30 \mathrm{kpc}$,

$a=G M_{\text {gal }} / R_{\text {gal }}^{2} \sim 0.8 \times 10^{-10} \mathrm{~m} / \mathrm{s}^{2}$

iv) For a typical cluster of galaxies, $M_{c}=10^{16}$ solar-masses and radius $R_{c}=3 \mathrm{M} \mathrm{pc}$,

$a=G M_{c} / R_{c}{ }^{2} \sim 10^{-10} \mathrm{~m} / \mathrm{s}^{2}$

v) Also, for the observable-universe as a whole, with a density of $10^{-29} \mathrm{grams} / \mathrm{cm}^{3}$ and radius $R_{0}=10^{28} \mathrm{~cm}$,

$a=H_{0} c=6.87 \times 10^{-10} \mathrm{~m} / \mathrm{s}^{2}$

vi) And the value of "critical acceleration of MOND, $a_{0} \sim 10^{-10} \mathrm{~m} / \mathrm{s}^{2}$

ii) As a supportive evidence for the above theory, let us look at the values of decelerations experienced by Pioneer-10, Pioneer-11, Galileo and Ulysses space-probes, (Anderson J.D. et al. 1998):

1) For Pioneer-10, $a=(8.09 \pm 0.2) \times 10^{-10} \mathrm{~m} / \mathrm{s}^{2}$

2) For Pioneer-11, $a=(8.56 \pm 0.15) \times 10^{-10} \mathrm{~m} / \mathrm{s}^{2}$

3) For Ulysses, $a=(12 \pm 3) \times 10^{-10} \mathrm{~m} / \mathrm{s}^{2}$

4) For Galileo, $a=(8.0 \pm 3) \times 10^{-10} \mathrm{~m} / \mathrm{s}^{2}$

All these decelerations are of the same order of magnitude as $H_{0} c$ $=6.87 \times 10^{-10} \mathrm{~m} / \mathrm{s}^{2}$; and match strikingly with the 'criticalacceleration' $a_{0}$ of MOND; an extremely rare-probability coincidence. Matching of four different decelerations of the spaceprobes, in spite of the differences in their mass, velocities and directions, is itself a striking coincidence; and its matching with the deceleration experienced by the 'cosmologically red-shifting photon' cannot be ignored by a scientific mind as a coincidence. So the atoms contained in the space-probes got decelerated due to the bit-by-bit decelerations caused by the virtual particles. This mechanism is testable in the laboratory. Or: b) By decay of a photon into a lighter photon and a particle of mass $h H_{0} / c^{2}$, at every wavelength traveled by the photon. Experimental detection of a new particle of such a small mass may be difficult but not impossible for the physicists. One of the ways of predicting a new particle is to calculate the missing energy, by applying the law of conservation of energy. The photon does loose a chunk of energy $h H_{0}$ while traveling every distance equal to its wavelength. This method suggests that a particle of mass $h H_{0} / c^{2}$ is likely to exist, based on the following argument: The strength ratio of electric forces and strong forces is $\sim \mathrm{e}^{2} / h c$, and we do find particles of the similar mass-ratio, namely, the mass-ratio of the electron and the pi-meson: $\left(m_{e} / m_{p i}\right) \sim\left(e^{2} / h c\right)$. Similarly, we can expect the particles with a mass-ratio: $\left.\left(h H_{0} / c^{2}\right) / m_{e}\right)=\left[\left(G m_{e} m_{\text {proton }}\right) /\right.$ $\left(e^{2}\right)$ ]. Interestingly the ratio $\left.\left(h H_{0} / c^{2}\right) / m_{e}\right)$ is indeed equal to the ratio $\left[\left(G m_{e} m_{\text {proton }}\right) /\left(e^{2}\right)\right]$ ! Therefore, our expectation of a particle of mass: $\left(h \mathrm{H}_{0} / c^{2}\right)$ is quite reasonable.

\section{Summary}

The straight-forward derivation presented here suggests that: the extra-galactic photons are decelerating at the rate $H_{0} c$ is an experimentally-observed fact. The gravitational acceleration at the "surface" of the virtual electrons, virtual positrons and virtual pimesons is also of the same order of magnitude as $H_{0} c$, so the gravitational field of the virtual particles bit-by-bit can keep on causing 'gravitational red-shift' in the extra-galactic photons. This reduction in the energy of the photons can explain the observation of 'cosmological red-shift'. This mechanism is testable in the laboratory. Possible alternative mechanism proposed here is: decay of the photon into a lighter photon and a new particle of mass: ( $h$ $\left.H_{0} / c^{2}\right)$.

\section{References}

[1] Tank Hasmukh K., (2013) "Wave-theoretical insight into the relativistic length-contraction and time-dilation of super-nova light-curves" Adv. Studies Theor. Phys., Vol. 7, no. 20, 971-976 http://dx.doi.org/10.12988/astp.2013.39102.

[2] Martin Lopez-Corredoira (2014) "Alcock-Paczynski Cosmolgical test" Astrophysical Journal Vol. 781(2) DOI:10.1088/0004-637X/781/2/96 http://dx.doi.org/10.1088/0004-637X/781/2/96.

[3] Ling Jun Wang, (2014) "On the Flatness of Spacetime" Physics Essays, Volume 27, No. 3

[4] Anderson, J. D., Laing, P. A., Lau, E. L., Liu, A. S., Nieto M. M., and Turyshev, S. G. (1998) "Indication, from Pioneer 10, 11, Galileo and Ulysses Data, of an Apparent Anomalous, Weak, Long-Range Acceleration". Phys. Rev. Letters. 81 2858-2861 [(Comment by Katz J.I: Phys. Rev. Lett. 83, 1892 (1999); Reply: Phys. Rev. Lett. 83, 1893 (1999)]. http://dx.doi.org/10.1103/PhysRevLett.81.2858.

[5] Sivaram, C. (1994) Astrophysics and Space science 215, 185-189 http://dx.doi.org/10.1007/BF00660076. 